\title{
Antioxidant activity of sesame seed lignans in sunflower and flaxseed oils
}

\author{
${ }^{1}$ Hadeel, S.Y., ${ }^{1}$ Khalida, S.A. and ${ }^{2, *}$ Walsh, M.K. \\ ${ }^{1}$ Department of Food Science, College of Agriculture, University of Baghdad, Iraq \\ ${ }^{2}$ Department of Nutrition, Dietetics, and Food Sciences, Utah State University, Logan, UT, USA
}

\begin{abstract}
Article history:
Received: 2 October 2019

Received in revised form: 19

November 2019

Accepted: 26 November

2019

Available Online: 22

December 2019
\end{abstract}

Keywords:

Sesame lignans,

Antioxidant activity,

Sesamin,

Sesamolin,

Sesamol

DOI:

https://doi.org/10.26656/fr.2017.4(3).331

\begin{abstract}
This study investigated the antioxidant activity of crude lignan extracts and purified lignans (sesamin, sesamolin, and sesamol) in sunflower and flaxseed oils. Lignan extracts were prepared from roasted sesame seed oil (LRSO) and unroasted sesame seed oil (LUSO). Additionally, the individual lignans were purified from both oils. The crude extracts and purified lignans were added at concentrations of $0.01,0.02$ and $0.03 \%$ to the oils and stored at 25 and $65^{\circ} \mathrm{C}$ over time and peroxide values and thiobarbituric acid values were measured. Each oil showed an increase in oxidation over time, with the samples stored at $65^{\circ} \mathrm{C}$ exhibiting accelerated oxidation. In general, LRSO showed higher antioxidant activity than LUSO and the antioxidant activity was similar to the antioxidant activity of butylated hydroxytoluene $(0.02 \%$ BHT) in both oils when used at concentrations of 0.02 and $0.03 \%$. Sesamol showed the highest antioxidant activity of each of the purified lignans followed by sesamin and sesamolin respectively. Crude and purified sesame lignans may have potential applications as natural antioxidants in food systems.
\end{abstract}

\section{Introduction}

Sesame oil (Sesamum indicum L.) contains many bioactive compounds such as phytosterols, tocopherols and lignans including sesamin, sesamolin and sesamol which have antioxidation properties. Sesame oil is high in oleic and linoleic fatty acids which are unsaturated. In fact, sesame oil shows high oxidative stability presumably due to the indigenous antioxidants (Hussain et al., 2018).

Lipid oxidation is a chemical reaction that occurs in oils that degrades the quality of the oil resulting in offflavors and odors generally referred to as rancidity. The initial stage in lipid oxidation is the generation of a free radical which can be induced by light, heat, metal ions and reactive oxygen species. In the presence of atmospheric oxygen, the primary oxidation products include peroxides, dienes and free fatty acids. These primary oxidative products are not stable and break down into secondary products which include aldehydes, ketones, alcohols and other high molecular weight compounds which are responsible for the rancid flavors and odors associated with oxidized lipids. Type 1 antioxidants donate a hydrogen to terminate oxidation and the most commonly used antioxidants in foods includes butylated hydroxy anisol (BHA), butylated hydroxytoluene (BHT), tocopherol and carotenoids. The synthetic antioxidants, BHA and BHT, are widely used due to their low cost but may not be accepted by consumers who would like clean ingredient labels.

Sunflower and flaxseed oils are considered healthy since they contain high levels of unsaturated fats. Unfortunately, oils high in unsaturated fatty acids are susceptible to oxidation and can have short shelf lives without the use of an antioxidant. Sunflower oil is rich in mono and polyunsaturated fatty acids including oleic acid (14-40\%) and linoleic acid (48-74\%) (Hussain et al., 2018). Flaxseed oil is rich in polyunsaturated fatty acids and low in saturated fatty acids. Flaxseed oil is also high in linolenic acid, 50-60\% (Mohanan et al., 2018). With the high level of unsaturated fatty acids, both of these oils are susceptible to oxidation.

Natural antioxidants from grape seed extracts (Poiana, 2012) potato peels, sugar beet pulp (Mohdaly et al., 2010) as well as sumac, thyme and sage (Basturk et al., 2017) have been investigated to prevent oxidation in unsaturated oils. There have been some studies on the effectiveness of crude extracts of sesame oil on the oxidative stability of unsaturated oils. Hussain et al. (2018) used three different extraction solvents, ethanol, methanol and water, to extract sesame lignans and then 
measured the effect of each extract on reducing the oxidation of sunflower oil. Konsoula and KiakipoulouKyriakides (2010) investigated the use of sesame seed extracts on the oxidation of oils (olive, sunflower and soybean) and found that the extracts were effective in protecting oils from oxidation and may have potential applications as a source of natural antioxidants for use in unsaturated oils. Lee and Choe (2006) investigated the effects of the individual sesame lignans, sesamol, sesamin, and sesamolin, on the oxidation of methyl linoleate. They found that the antioxidant activity of sesamol was significantly higher than that of sesamin and sesamolin.

Although there have been studies on the antioxidant properties of sesame lignans in food-grade oils, there have been no studies that compared the crude lignan extracts from unroasted and roasted sesame seeds and the individually purified lignans on the oxidative stability of food oils. This study investigated the effects of roasted sesame seeds on the quantity and antioxidant activity of lignans compared to lignans from unroasted sesame seed. Crude lignan extracts from both roasted and unroasted sesame seeds and individually purified sesamol, sesamin and sesaminol were added to sunflower and flaxseed oils at $25^{\circ} \mathrm{C}$ and $65^{\circ} \mathrm{C}$ and the oxidative stability of the oils was compared to the same oils containing BHT over storage time.

\section{Materials and methods}

\subsection{Sesame seed roasting and oil extraction}

Sesame seeds (Sesame indicum L.) were obtained from a local market in Baghdad, Iraq. Impurities (dust and sand) were removed using a $2.4 \mathrm{~mm}$ diameter sieve and the seeds were washed with water. Seeds were then dried at $40^{\circ} \mathrm{C}$. Seeds were roasted at $220^{\circ} \mathrm{C}$ for $30 \mathrm{mins}$ in glass dishes in a muffle furnace. Separate dishes were roasted at different times and pooled for oil extraction.

Sesame oil was extracted from both unroasted (USO) and roasted (RSO) seeds using hexane. Sesame seeds, $50 \mathrm{~g}$ were milled in a coffee grinder and mixed with hexane (a 5-fold volume) and placed in an ultrasonic bath at $25^{\circ} \mathrm{C}$ for 30 mins as described by Konsoula and Kiakipoulou-Kyriakides, 2010. The hexane was decanted and this process was repeated twice using fresh hexane. The hexane fraction was filtered and the hexane in the combined filtrates was evaporated under vacuum at $40^{\circ}$ C. The remaining oil was stored in a freezer at $-20^{\circ} \mathrm{C}$. The percentage of oil extracted was calculated by dividing the weight of the oil by the total weight of the starting seeds.

\subsection{Extraction of lignans from sesame oil}

The lignans in the sesame oil were extracted with methanol as previously described (Reshma et al., 2010). Briefly, the oil was mixed with 2 volumes of methanol and placed in an extraction vessel consisting of a two necked flask equipped with a reflux condenser and thermometer. The sample was stirred continuously for 10 mins at $70^{\circ} \mathrm{C}$ with a magnetic stirrer then the sample was transferred to a separatory funnel. After $15 \mathrm{~min}$, the methanol layer was separated from the oil and the oil was again mixed with methanol for a total of 5 methanol extractions. All methanol samples were pooled and the methanol allowed to evaporate in a fume hood. The dried methanol extracts were termed lignans from RSO (LRSO) and lignans from USO (LUSO). The quantity of sesamin, sesamolin and sesamol in RSO and USO extracts were determined via HPLC as described below.

Sesamin and sesamolin were separated and crystallized from RSO according to the method described by Lee and Choe (2006) and Hemalatha (2007). Oil, $60 \mathrm{~g}$, was dissolved in $900 \mathrm{~mL}$ of acetone and cooled for $24 \mathrm{hrs}$ at $-50^{\circ} \mathrm{C}$ and filtered through a Buchner funnel and Whatman no. 42 filter paper to remove crystallized triacylglycerols. The acetone was eliminated by evaporation under vacuum at $40^{\circ} \mathrm{C}$ using a rotary evaporator, which resulted in a yellow oil. This was mixed with iso-octane $(1: 8 \mathrm{v} / \mathrm{v})$ and left at $4^{\circ} \mathrm{C}$ for 5 days to precipitate the sesamin crystals. Iso-octane was evaporated under vacuum and the remaining sample was saponified with $5 \%$ ethanolic potassium hydroxide (25 $\mathrm{mL}$ ) for $1 \mathrm{hr}$, then $100 \mathrm{~mL}$ of water was added and the soap solution was extracted with diethyl ether $(60 \mathrm{~mL}) 3$ times. The ether was removed by evaporation at room temperature and the remaining sample was dissolved in chloroform $(1 \mathrm{~mL})$ and then petroleum ether was added until the onset of cloudiness. Sesamolin crystallized from the solution. Both sesamin and sesamolin were recrystallized in ethanol.

Sesamol was isolated from sesame oil according to Reshma et al. (2010) with modifications. The RSO, 100 $\mathrm{g}$, was mixed with water and $\mathrm{n}$-hexane in ratios of $1: 1: 0.05 \mathrm{w} / \mathrm{v} / \mathrm{v}$ into a separatory funnel and left for 15 min to separate into two layers. The oil and the water phases were separated. The residual oil from the first extraction was extracted a second time with 1 volume of water and this water extraction was repeated 3 more times. All water fractions were pooled and concentrated using a rotary evaporator. The dried product was resuspended and crystallized in ethanol.

The purity and quantity of lignans were determined via HPLC using the method described by Reshma et al. (2010). Lignan standards (Sigma-Aldrich, USA) were 
dissolved in methanol and separated using a Shimadzu HPLC system (model LC-200 AHT) equipped with a C18 column ( $4.6 \mathrm{~mm} \times 150 \mathrm{~mm}$, id 5 micron, Waters, Co. Milford MA USA). The mobile phase was $70 \%$ methanol and $30 \%$ water at a flow rate of $0.5 \mathrm{~mL} / \mathrm{min}$ and lignans were detected at $290 \mathrm{~nm}$. Purified lignans were analyzed and their concentration and purity determined as compared to the standards.

\subsection{Antioxidant properties of lignans in oil}

Food grade sunflower oil and flaxseed oil were purchased from Sigma-Aldrich (USA). Stock solutions (10\% in ethanol) of LRSO and LUSO, as well as the purified lignans, were prepared and mixed into the oils at concentrations of 0, 0.01, 0.02 and $0.03 \%$. BHT (SigmaAldrich, USA) stock solution of $10 \%$ in ethanol was prepared and added to oils for a final concentration of $0.02 \%$. The samples were stored in amber bottles and kept at 25 or $65^{\circ} \mathrm{C}$ for up to 75 days.

The oxidative stability of the oils was measured by determining peroxide value (PV) and thiobarbituric acid values (TBA). The PV was measured according to the AOCS method No 19.90 (AOCS, 2001). Samples, $5 \mathrm{~g}$, were weighed into a $250 \mathrm{~mL}$ flask, $30 \mathrm{~mL}$ of acetic acid/ chloroform solution $(6: 10 \mathrm{v} / \mathrm{v})$ was added and mixed thoroughly. Potassium iodide (saturated, $10 \mathrm{~g} \mathrm{KI}$ in 6 $\mathrm{mL}$ water) was added with stirring for $1 \mathrm{~min}$ followed by the addition of $30 \mathrm{~mL}$ distilled water and $0.5 \mathrm{~mL}$ of a $1 \%$ starch solution (corn, Sigma-Aldrich, USA). The sample was titrated with $0.1 \mathrm{~N}$ sodium thiosulfate until the solution turned from a blue to light yellow color. The amount of thiosulfate solution required for the color change was noted and the PV was calculated using the following equation: Peroxide value $\left(\right.$ meq $\mathrm{O}_{2} / \mathrm{kg}$ oil $)=$ $\mathrm{mL}$ of titrant $\times$ normality of titrant $\times 1000 /$ sample $\mathrm{g}$ weight.

The TBA value was determined according to the AOAC method (2017). Oil, $20 \mathrm{mg}$ was weighed into a 25 $\mathrm{mL}$ volumetric flask and 1-butanol was added to reach $25 \mathrm{~mL}$. This sample, $5 \mathrm{~mL}$, was mixed with $5 \mathrm{~mL}$ of the thiobarbituric acid solution (13.9 $\mathrm{mM}$ in 1-butanol) and vortexed. The samples were heated in a water bath at $95^{\circ}$ $\mathrm{C}$ for $2 \mathrm{hrs}$, then cooled with water to $25^{\circ} \mathrm{C}$. The absorbance at $530 \mathrm{~nm}$ was measured in a spectrophotometer (SCO-TECH, Germany) and compared to a blank solution that did not contain oil. TBA was calculated as mg malonaldehyde $/ \mathrm{kg}$ oil $=50 \mathrm{x}$ (absorbance of the sample - absorbance of blank)/ sample g weight.

\subsection{Statistical analysis}

All assays were conducted in duplicate and replicated three times. The Statistical Analysis System
(SAS 2012) was used to analyze the data and the least significant difference (LSD) was determined.

\section{Results}

\subsection{Purification of lignans from sesame oil}

Oil from RSO and USO was extracted and the yields were $44.00 \%$ and $53.20 \%$ for RSO and USO respectively. The yield of sesamin, sesamolin, and sesamol found in RSO and USO are tabulated in Table 1. The lignans present in RSO were $60 \%$ sesamin, $35.5 \%$ sesamolin and $4 \%$ sesamol. The lignans present in USO were $61 \%$ sesamin and $39 \%$ sesamolin. Sesamol is formed from the breakdown of sesamolin which occurs during roasting, therefore the USO did not contain any sesamol. These results are similar to the results obtained by Reshma et al. (2010) with methanol extracts of unroasted sesame seed oil. They showed that the concentration of sesamin in the lignan extract was $65 \%$

Table 1. Lignan content in sesame seed oil

\begin{tabular}{lcc}
\hline & RSO $(\mathrm{mg} / 100 \mathrm{mg}$ oil $)$ & USO $(\mathrm{mg} / 100 \mathrm{mg}$ oil $)$ \\
\hline Total Lignans & 0.2 & 0.18 \\
Sesamin & 0.119 & 0.11 \\
Sesamolin & 0.071 & 0.071 \\
Sesamol & 0.008 & 0 \\
\hline
\end{tabular}

RSO: Roasted sesame seed oil, USO: Unroasted sesame seed oil

and the amount of sesamolin in the extract was $27.8 \%$.

\subsection{Oxidation in sunflower oil}

Sunflower oil is prone to oxidation due to its high concentration of polyunsaturated fatty acids. Sunflower oil was treated with crude lignin extracts from roasted (LRSO) and unroasted (LUSO) sesame seed oils at concentrations ranging from 0.01 to $0.03 \%$. Sunflower oil was also treated with $0.02 \%$ BHT (a common use level in oils) (Poiana, 2012) and untreated as control. Peroxide values (PV) measure the primary products of lipid oxidation, namely hydroperoxides. The results of the PVs in sunflower oil stored at both 25 and $65^{\circ} \mathrm{C}$ is tabulated in Table 2. In general, with an increase in time and at $65^{\circ} \mathrm{C}$ (accelerated oxidation conditions), there was an increase in the PVs for each treatment, with the control without antioxidant showing significantly higher PVs than all other treatments by the end of the testing. For samples stored at $65^{\circ} \mathrm{C}$, at days 12 and 16 , both the LRSO and LUSO treatments at 0.02 and $0.03 \%$ were similar to the BHT treated samples. In general, the LRSO PVs were lower than both the LUSO and BHT but not statistically. At $25^{\circ} \mathrm{C}$ and at all times, the LRSO and LUSO PVs at 0.02 and $0.03 \%$ were not statistically different than the BHT samples and all were lower than without antioxidant. Again, the LRSO PVs were lower 
Table 2. Effect of roasted and unroasted sesame oil lignan extracts on the peroxide values of sunflower oil stored at 65 and $25^{\circ} \mathrm{C}$

\begin{tabular}{|c|c|c|c|c|c|c|}
\hline \multirow{2}{*}{$\begin{array}{l}\text { Days } \\
65^{\circ} \mathrm{C} \\
\end{array}$} & \multirow[b]{2}{*}{ Antioxidant (\%) } & \multirow[b]{2}{*}{$\mathrm{LRSO}^{1}$} & \multicolumn{4}{|c|}{ Peroxide Values (meq $\mathrm{O}_{2} / \mathrm{kg}$ oil) } \\
\hline & & & $\mathrm{LUSO}^{2}$ & $\operatorname{BHT}^{3}(0.02 \%)$ & Without antioxidant & $\mathrm{LSD}^{4}$ \\
\hline 0 & 0 & 3.95 & 3.95 & 3.95 & 3.95 & \\
\hline \multirow{3}{*}{4} & 0.01 & 18.22 & 18.3 & \multirow{3}{*}{11.74} & \multirow{3}{*}{12.7} & 3.38 \\
\hline & 0.02 & 11.88 & 13.9 & & & 2.87 \\
\hline & 0.03 & 11.44 & 15.46 & & & 3.57 \\
\hline \multirow{3}{*}{12} & 0.01 & 30.7 & 43.92 & \multirow{3}{*}{22.72} & \multirow{3}{*}{46.62} & 4.72 \\
\hline & 0.02 & 23.46 & 24.12 & & & 4.09 \\
\hline & 0.03 & 19.56 & 21.48 & & & 4.21 \\
\hline \multirow{3}{*}{16} & 0.01 & 49.28 & 53.88 & \multirow{3}{*}{36.68} & \multirow{3}{*}{60.6} & 4.58 \\
\hline & 0.02 & 38.26 & 40.46 & & & 5.64 \\
\hline & 0.03 & 33.28 & 34.76 & & & 5.91 \\
\hline \multirow[t]{2}{*}{ LSD Value } & & 7.27 & 5.92 & 5.18 & 7.61 & \\
\hline & \multicolumn{6}{|c|}{ Peroxide Values (meq $\mathrm{O}_{2} / \mathrm{kg}$ oil) } \\
\hline $\begin{array}{l}\text { Days } \\
25^{\circ} \mathrm{C}\end{array}$ & Antioxidant (\%) & $\mathrm{LRSO}^{1}$ & $\mathrm{LUSO}^{2}$ & $\mathrm{BHT}^{3}(0.02 \%)$ & Without antioxidant & $\mathrm{LSD}^{4}$ \\
\hline 0 & 0 & 3.95 & 3.95 & 3.95 & 3.95 & \\
\hline \multirow{3}{*}{15} & 0.01 & 4.73 & 7.92 & \multirow{3}{*}{4.91} & \multirow{3}{*}{9.5} & 1.26 \\
\hline & 0.02 & 4.35 & 4.77 & & & 1.33 \\
\hline & 0.03 & 3.96 & 3.99 & & & 2.56 \\
\hline \multirow{3}{*}{45} & 0.01 & 16.93 & 18.56 & \multirow{3}{*}{13.98} & \multirow{3}{*}{22.84} & 4.52 \\
\hline & 0.02 & 14.83 & 15.33 & & & 4.25 \\
\hline & 0.03 & 11.95 & 13.99 & & & 4.88 \\
\hline \multirow{3}{*}{75} & 0.01 & 27.6 & 31.54 & \multirow{3}{*}{25.16} & \multirow{3}{*}{35.37} & 4.65 \\
\hline & 0.02 & 25.16 & 29.86 & & & 5.61 \\
\hline & 0.03 & 23.62 & 28.94 & & & 4.68 \\
\hline LSD Value & & 4.82 & 5.02 & 4.19 & 6.44 & \\
\hline
\end{tabular}

${ }^{1}$ LRSO, Lignin extracts from roasted sesame seeds, ${ }^{2}$ LUSO, Lignin extracts from unroasted sesame seeds, ${ }^{3} \mathrm{BHT}$, butylated hydroxytoluene, ${ }^{4} \mathrm{LSD}$, least significant difference, if the means differ by the LSD number the values are significantly different.

Table 3. Effect of purified lignans on the peroxide values of sunflower oil stored at 65 and $25^{\circ} \mathrm{C}$

\begin{tabular}{|c|c|c|c|c|c|c|c|}
\hline \multirow{2}{*}{ Days $65^{\circ} \mathrm{C}$} & \multirow[b]{2}{*}{ Antioxidant (\%) } & \multicolumn{6}{|c|}{ Peroxide Values (meq $\mathrm{O}_{2} / \mathrm{kg}$ oil) } \\
\hline & & Sesamol & Sesamin & Sesamolin & $\operatorname{BHT}^{1}(0.02 \%)$ & Without antioxidant & $\mathrm{LSD}^{2}$ \\
\hline 0 & 0 & 3.95 & 3.95 & 3.95 & 3.95 & 3.95 & \\
\hline \multirow{3}{*}{4} & 0.01 & 11.76 & 17.2 & 19.58 & \multirow{3}{*}{11.74} & \multirow{3}{*}{20.7} & 2.55 \\
\hline & 0.02 & 10.96 & 11.91 & 16.66 & & & 2.84 \\
\hline & 0.03 & 9.78 & 11.72 & 12.16 & & & 2.75 \\
\hline \multirow{3}{*}{12} & 0.01 & 26.92 & 35.08 & 46.68 & \multirow{3}{*}{22.72} & \multirow{3}{*}{46.62} & 4.61 \\
\hline & 0.02 & 22.68 & 23.5 & 27.36 & & & 4.53 \\
\hline & 0.03 & 20.44 & 20.74 & 22.66 & & & 4.66 \\
\hline \multirow{3}{*}{16} & 0.01 & 43.4 & 46.68 & 56.82 & \multirow{3}{*}{36.68} & \multirow{3}{*}{60.6} & 5.02 \\
\hline & 0.02 & 36.12 & 36.88 & 55.96 & & & 4.83 \\
\hline & 0.03 & 22.3 & 33.44 & 34.02 & & & 4.77 \\
\hline \multirow[t]{2}{*}{ LSD Value } & & 5.66 & 5.29 & 6.76 & 5.18 & 7.61 & \\
\hline & & \multicolumn{6}{|c|}{ Peroxide Values (meq $\mathrm{O}_{2} / \mathrm{kg}$ oil) } \\
\hline Days $25^{\circ} \mathrm{C}$ & Antioxidant (\%) & Sesamol & Sesamin & Sesamolin & $\mathrm{BHT}^{1}(0.02 \%)$ & Without antioxidant & $\mathrm{LSD}^{2}$ \\
\hline 0 & 0 & 3.95 & 3.95 & 3.95 & 3.95 & 3.95 & \\
\hline \multirow{3}{*}{15} & 0.01 & 4.37 & 5.16 & 8.77 & \multirow{3}{*}{4.91} & \multirow{3}{*}{9.5} & 2.36 \\
\hline & 0.02 & 4.32 & 4.73 & 4.78 & & & 2.77 \\
\hline & 0.03 & 3.89 & 3.83 & 3.94 & & & 2.64 \\
\hline \multirow{3}{*}{45} & 0.01 & 15.65 & 17.12 & 21.62 & \multirow{3}{*}{13.98} & \multirow{3}{*}{22.84} & 4.09 \\
\hline & 0.02 & 13.83 & 15.21 & 16.28 & & & 4.21 \\
\hline & 0.03 & 9.38 & 13.98 & 13.13 & & & 3.56 \\
\hline \multirow{3}{*}{75} & 0.01 & 27.48 & 30.88 & 33.06 & \multirow{3}{*}{25.16} & \multirow{3}{*}{35.37} & 3.25 \\
\hline & 0.02 & 24.68 & 27.32 & 31.07 & & & 3.08 \\
\hline & 0.03 & 17.76 & 24.68 & 29.86 & & & 4.66 \\
\hline LSD Value & & 4.79 & 4.63 & 5.62 & 4.19 & 6.44 & \\
\hline
\end{tabular}

${ }^{1}$ BHT, butylated hydroxytoluene, ${ }^{2}$ LSD, least significant difference, if the means differ by the LSD number the values are significantly different. 
than the LUSO and BHT PVs, but this was not a statistical difference. Additionally, samples treated with $0.01 \%$ of the lignans showed statistically higher PVs over time, with the values on day 16 being statistically different, therefore the antioxidant effect of lignans was concentration-dependent. Lignans from USO and RSO used at $0.01 \%$ were not as effective as when used at higher concentrations. Lignans from RSO had higher antioxidant activity than lignans from USO and BHT.

The increase in PVs of the control sunflower oil at day 16 was $1434 \%$ compared to $742 \%, 780 \%$ and $780 \%$ with treatments of LRSO $(0.03 \%)$, LUSO $(0.03 \%)$ and BHT $(0.02 \%)$ respectively at $65^{\circ} \mathrm{C}$. While the increase in PVs of the control sunflower oil at $25^{\circ} \mathrm{C}$ at approximately the same storage time (day 15 ) was $140 \%$, compared to $24 \%$ with BHT and no increase in oxidation with LRSO or LUSO. Therefore, the storage temperature significantly increased the oxidation rate of sunflower oil.

The effects of each individually purified lignans on the PVs in sunflower oil stored at both 25 and $65^{\circ} \mathrm{C}$ are tabulated in Table 3. There was an increase in PVs over storage time for both temperatures with the control sample having the highest PVs. At $65^{\circ} \mathrm{C}$ and at day 16 with $0.02 \%$ sesamol and sesamin, the PVs were not statistically different from the BHT samples. However, the sesamol samples showed a significantly lower PV at $0.03 \%$ from all other treatments at day 16 . This same trend was observed in samples stored at $25^{\circ} \mathrm{C}$ where the sesamol samples showed significantly lower PVs when used at $0.03 \%$ than all other treatments. In general, samples treated with sesamolin showed higher PVs than samples treated with sesamol, sesamin and BHT although there was not always a statistical difference. Samples treated with $0.01 \%$ of lignans showed the highest PVs of all concentrations tested. The percentage increase in PVs at $65^{\circ} \mathrm{C}$ and at day 16 was $465 \%$ for samples containing sesamol which is less than the percentage increase in samples at the same conditions containing sesamin (746\%), sesamolin (761\%) and BHT $(828 \%)$.

To confirm the results obtained in Tables 2 and 3, the thiobarbituric acid (TBA) values of the oils were also measured. The TBA test measures secondary oxidative products, namely malonaldehyde generally formed from hydroperoxides. The TBA values of sunflower samples stored at 25 and $65^{\circ} \mathrm{C}$ over time was measured and the values are tabulated in Table 4 . In general, there were higher TBA values at $65^{\circ} \mathrm{C}$ compared to $25^{\circ} \mathrm{C}$ and higher values with an increase in time and a decrease in lignan concentration. Also, samples treated with LRSO tended to have lower TBA values than LUSO treated samples at all treatments. Over the storage time, all LRSO, LUSO and BHT treatments were lower than the control. The effect of the addition of individual sesame lignans on the TBA values of sunflower oil stored at 65 and $25^{\circ} \mathrm{C}$ over time is tabulated in Table 5. As with the PVs, the controls showed the highest TBA values overtime at both storage temperatures. Generally, samples containing sesamol at concentrations of 0.02 and $0.03 \%$ showed the lowest TBA values, lower than BHT treated samples on each day tested. Sesamin was more effective at the highest concentration only, while sesamolin was not as effective as the other lignans or BHT at any concentration.

\subsection{Oxidation in flaxseed oil}

The antioxidant effect as measured by the PV of sesame oil lignans in flaxseed oil is tabulated in Table 6. As with the sunflower oil study, all samples showed an increase in PVs with an increase in time and at $65^{\circ} \mathrm{C}$ compared to $25^{\circ} \mathrm{C}$. At $65^{\circ} \mathrm{C}$ and on day 16 , flaxseed oil containing 0.02 and $0.03 \%$ LRSO showed the lowest PV, which were significantly lower than flaxseed oil containing BHT. Flaxseed oil containing $0.03 \%$ LUSO at the same time point were statistically lower than the oil containing BHT. At $25^{\circ} \mathrm{C}$, flaxseed oil containing LRSO and LUSO were lower than the BHT treated samples, but there was not a statistical difference. All treatments were lower than the sample without antioxidant. The percentage increase in PVs for samples containing $0.03 \%$ LRSO (2457\%), LUSO (2502\%) and BHT $(3520 \%)$ were much lower than the control sample which had a $4814 \%$ increase at $65^{\circ} \mathrm{C}$ at day 16 . Additionally, the flaxseed oil had lower initial PVs than the initial sunflower oil samples but the percentage increase in PVs for flaxseed oil at $65^{\circ} \mathrm{C}$ at day 16 was 3.5 times higher than with sunflower oil. Therefore, flaxseed oil is more susceptible to oxidation at $65^{\circ} \mathrm{C}$ than sunflower oil.

Table 7 tabulates the PVs for flaxseed oil samples treated with individual lignans as compared to BHT and without antioxidant. As seen in the sunflower oil samples, samples containing sesamol and sesamin had lower PVs compared to the BHT sample at all concentrations used at $65^{\circ}$ at day 16 . Samples containing sesamolin $(0.03 \%)$ under the same conditions had lower PVs than BHT. A similar trend is seen at $25^{\circ}$ $\mathrm{C}$ where sesamol PVs were lower than the BHT treated sample at day 75 and at concentrations of 0.02 and $0.03 \%$. At both temperatures, sesamolin was not as effective as sesamol, sesamin or BHT with some PVs similar to the control without antioxidant. The percentage increase in PVs with sesamol at $0.03 \%$ was $2694 \%$ compared to $3139 \%$ and $3300 \%$ with sesamin 
Table 4. Effect of roasted and unroasted sesame oil lignan extracts on the thiobarbituric acid (TBA) values of sunflower oil stored at 65 and $25^{\circ} \mathrm{C}$

\begin{tabular}{|c|c|c|c|c|c|c|}
\hline & & & TB & (mg/ MDA/kg & iil) & \\
\hline $\begin{array}{l}\text { Days } \\
65^{\circ} \mathrm{C}\end{array}$ & Antioxidant (\%) & $\mathrm{LRSO}^{1}$ & $\mathrm{LUSO}^{2}$ & $\operatorname{BHT}^{3}(0.02 \%)$ & Without antioxidant & $\mathrm{LSD}^{4}$ \\
\hline 0 & 0 & 0.105 & 0.105 & 0.105 & 0.105 & \\
\hline & 0.01 & 0.314 & 0.369 & & & 0.266 \\
\hline 4 & 0.02 & 0.260 & 0.262 & 0.183 & 0.560 & 0.285 \\
\hline & 0.03 & 0.166 & 0.227 & & & 0.261 \\
\hline & 0.01 & 0.445 & 0.826 & & & 0.202 \\
\hline 12 & 0.02 & 0.388 & 0.581 & 0.375 & 0.988 & 0.241 \\
\hline & 0.03 & 0.235 & 0.396 & & & 0.276 \\
\hline & 0.01 & 0.976 & 0.855 & & & 0.283 \\
\hline 16 & 0.02 & 0.742 & 0.685 & 0.418 & 1.039 & 0.252 \\
\hline & 0.03 & 0.315 & 0.482 & & & 0.248 \\
\hline LSD Value & & 0.267 & 0.422 & 0.296 & 0.507 & \\
\hline & & & TB & (mg/ MDA/kg & iil) & \\
\hline $\begin{array}{l}\text { Days } \\
25^{\circ} \mathrm{C}\end{array}$ & Antioxidant (\%) & $\mathrm{LRSO}^{1}$ & $\mathrm{LUSO}^{2}$ & $\mathrm{BHT}^{3}(0.02 \%)$ & Without antioxidant & $\mathrm{LSD}^{4}$ \\
\hline 0 & 0 & 0.105 & 0.105 & 0.105 & 0.105 & \\
\hline & 0.01 & 0.296 & 0.345 & & & 0.165 \\
\hline 15 & 0.02 & 0.166 & 0.201 & 0.193 & 0.328 & 0.172 \\
\hline & 0.03 & 0.0123 & 0.152 & & & 0.104 \\
\hline & 0.01 & 0.309 & 0.592 & & & 0.138 \\
\hline 45 & 0.02 & 0.282 & 0.331 & 0.283 & 0.855 & 0.187 \\
\hline & 0.03 & 0.215 & 0.310 & & & 0.161 \\
\hline & 0.01 & 0.453 & 0.656 & & & 0.158 \\
\hline 75 & 0.02 & 0.352 & 0.399 & 0.325 & 0.658 & 0.194 \\
\hline & 0.03 & 0.218 & 0.377 & & & 0.108 \\
\hline LSD Value & & 0.108 & 0.142 & 0.088 & 0.271 & \\
\hline
\end{tabular}

${ }^{1}$ LRSO, Lignin extracts from roasted sesame seeds, ${ }^{2}$ LUSO, Lignin extracts from unroasted sesame seeds, ${ }^{3} \mathrm{BHT}$, butylated hydroxytoluene, ${ }^{4} \mathrm{LSD}$, least significant difference, if the means differ by the LSD number the values are significantly different.

Table 5. Effect of purified lignans on the thiobarbituric acid (TBA) values of sunflower oil stored at 65 and $25^{\circ} \mathrm{C}$.

\begin{tabular}{|c|c|c|c|c|c|c|c|}
\hline \multirow{2}{*}{ Days $65^{\circ} \mathrm{C}$} & \multirow[b]{2}{*}{ Antioxidant (\%) } & \multicolumn{6}{|c|}{ TBA (mg/ MDA/kg oil) } \\
\hline & & Sesamol & Sesamin & Sesamolin & $\operatorname{BHT}^{1}(0.02 \%)$ & Without antioxidant & $\mathrm{LSD}^{2}$ \\
\hline 0 & 0 & 0.105 & 0.105 & 0.105 & 0.105 & 0.105 & \\
\hline \multirow{3}{*}{4} & 0.01 & 0.214 & 0.394 & 0.501 & \multirow{3}{*}{0.183} & \multirow{3}{*}{0.560} & 0.264 \\
\hline & 0.02 & 0.174 & 0.196 & 0.205 & & & 0.288 \\
\hline & 0.03 & 0.118 & 0.136 & 0.175 & & & 0.276 \\
\hline \multirow{3}{*}{12} & 0.01 & 0.395 & 0.605 & 0.743 & \multirow{3}{*}{0.375} & \multirow{3}{*}{0.988} & 0.267 \\
\hline & 0.02 & 0.346 & 0.407 & 0.437 & & & 0.217 \\
\hline & 0.03 & 0.215 & 0.282 & 0.293 & & & 0.252 \\
\hline \multirow{3}{*}{16} & 0.01 & 0.625 & 0.720 & 0.807 & \multirow{3}{*}{0.418} & \multirow{3}{*}{1.039} & 0.282 \\
\hline & 0.02 & 0.402 & 0.488 & 0.619 & & & 0.224 \\
\hline & 0.03 & 0.282 & 0.337 & 0.429 & & & 0.296 \\
\hline \multirow[t]{2}{*}{ LSD Value } & & 0.237 & 0.446 & 0.485 & 0.296 & 0.507 & \\
\hline & & \multicolumn{6}{|c|}{ TBA (mg/ MDA/kg oil) } \\
\hline Days $25^{\circ} \mathrm{C}$ & Antioxidant (\%) & Sesamol & Sesamin & Sesamolin & $\mathrm{BHT}^{1}(0.02 \%)$ & Without antioxidant & $\mathrm{LSD}^{2}$ \\
\hline 0 & 0 & 0.105 & 0.105 & 0.105 & 0.105 & 0.105 & \\
\hline \multirow{3}{*}{15} & 0.01 & 0.253 & 0.328 & 0.377 & \multirow{3}{*}{0.193} & \multirow{3}{*}{0.328} & 0.181 \\
\hline & 0.02 & 0.152 & 0.164 & 0.219 & & & 0.173 \\
\hline & 0.03 & 0.108 & 0.136 & 0.159 & & & 0.235 \\
\hline \multirow{3}{*}{45} & 0.01 & 0.346 & 0.439 & 0.463 & \multirow{3}{*}{0.283} & \multirow{3}{*}{0.855} & 0.278 \\
\hline & 0.02 & 0.280 & 0.310 & 0.360 & & & 0.281 \\
\hline & 0.03 & 0.193 & 0.202 & 0.299 & & & 0.202 \\
\hline \multirow{3}{*}{75} & 0.01 & 0.378 & 0.464 & 0.702 & \multirow{3}{*}{0.325} & \multirow{3}{*}{0.958} & 0.197 \\
\hline & 0.02 & 0.312 & 0.443 & 0.518 & & & 0.215 \\
\hline & 0.03 & 0.215 & 0.231 & 0.380 & & & 0.252 \\
\hline LSD Value & & 0.0116 & 0.173 & 0.209 & 0.088 & 0.271 & \\
\hline
\end{tabular}

${ }^{1}$ BHT, butylated hydroxytoluene, ${ }^{2}$ LSD, least significant difference, if the means differ by the LSD number the values are significantly different. 
Table 6. Effect of roasted and unroasted sesame oil lignan extracts on the peroxide values of flaxseed oil stored at 65 and $25^{\circ} \mathrm{C}$

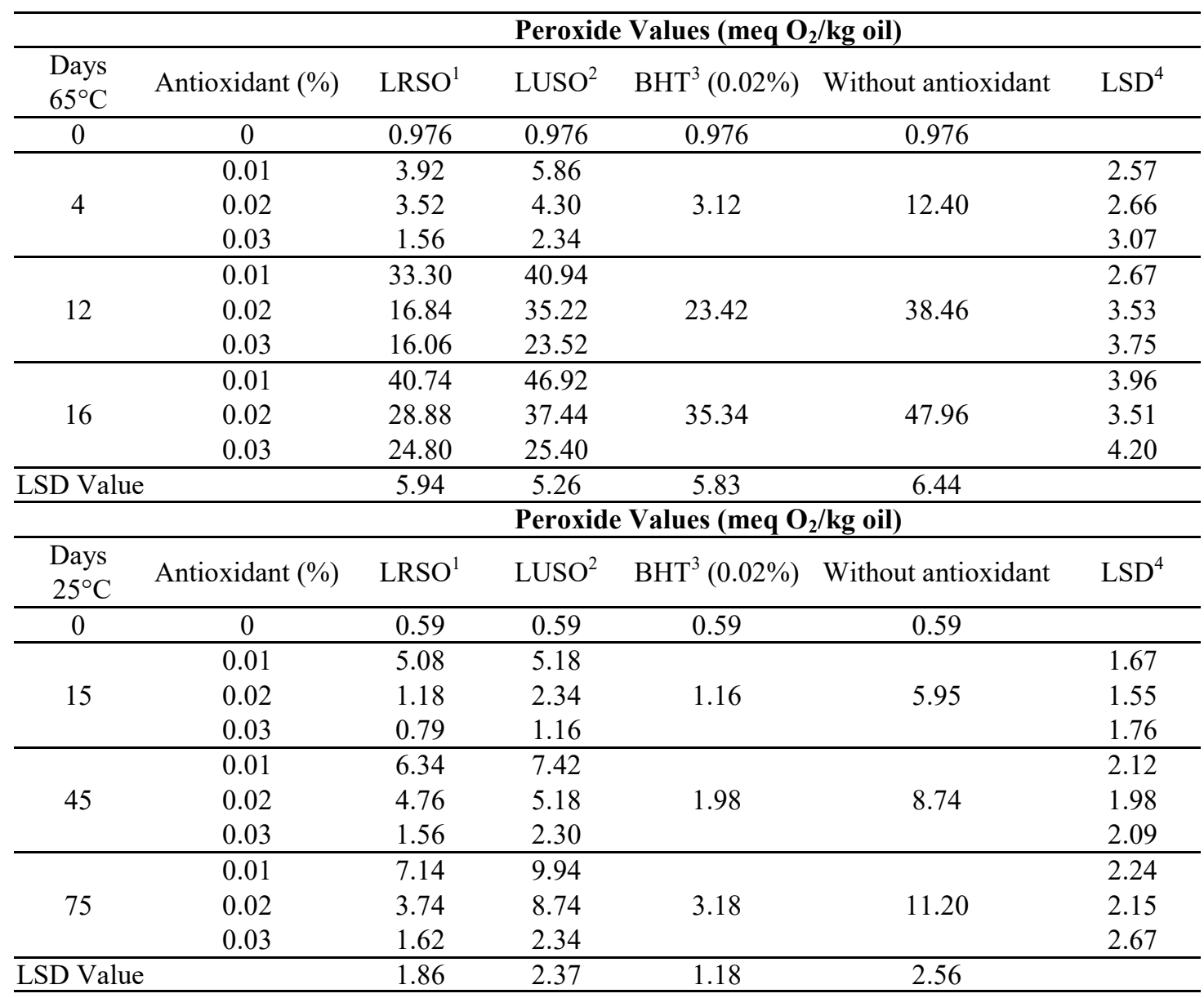

${ }^{1}$ LRSO, Lignin extracts from roasted sesame seeds, ${ }^{2}$ LUSO, Lignin extracts from unroasted sesame seeds, ${ }^{3}$ BHT, butylated hydroxytoluene, ${ }^{4} \mathrm{LSD}$, least significant difference, if the means differ by the LSD number the values are significantly different.

Table 7. Effect of purified lignans on the peroxide values of flaxseed oil stored at 65 and $25^{\circ} \mathrm{C}$

\begin{tabular}{|c|c|c|c|c|c|c|c|}
\hline \multirow{2}{*}{ Days $65^{\circ} \mathrm{C}$} & \multirow[b]{2}{*}{ Antioxidant (\%) } & \multicolumn{6}{|c|}{ Peroxide Values (meq $\mathrm{O}_{2} / \mathrm{kg}$ oil) } \\
\hline & & Sesamol & Sesamin & Sesamolin & $\operatorname{BHT}^{1}(0.02 \%)$ & Without antioxidant & $\mathrm{LSD}^{2}$ \\
\hline 0 & 0 & 0.98 & 0.98 & 0.98 & 0.98 & 0.98 & \\
\hline \multirow{3}{*}{4} & 0.01 & 4.70 & 6.26 & 12.14 & \multirow{3}{*}{3.12} & \multirow{3}{*}{12.4} & 2.67 \\
\hline & 0.02 & 1.98 & 5.08 & 10.18 & & & 2.55 \\
\hline & 0.03 & 0.80 & 2.34 & 3.56 & & & 2.72 \\
\hline \multirow{3}{*}{12} & 0.01 & 21.92 & 35.54 & 34.83 & \multirow{3}{*}{23.42} & \multirow{3}{*}{38.46} & 3.53 \\
\hline & 0.02 & 16.80 & 29.70 & 31.16 & & & 3.66 \\
\hline & 0.03 & 12.52 & 15.66 & 22.82 & & & 4.09 \\
\hline \multirow{3}{*}{16} & 0.01 & 32.66 & 34.54 & 40.12 & \multirow{3}{*}{35.34} & \multirow{3}{*}{47.96} & 3.86 \\
\hline & 0.02 & 26.70 & 31.38 & 32.68 & & & 3.91 \\
\hline & 0.03 & 27.10 & 31.42 & 32.98 & & & 4.77 \\
\hline \multirow[t]{2}{*}{ LSD Value } & & 5.68 & 5.07 & 6.31 & 5.83 & 6.44 & \\
\hline & & \multicolumn{6}{|c|}{ Peroxide Values (meq $\mathrm{O}_{2} / \mathrm{kg}$ oil) } \\
\hline Days $25^{\circ} \mathrm{C}$ & Antioxidant $(\%)$ & Sesamol & Sesamin & Sesamolin & $\operatorname{BHT}^{1}(0.02 \%)$ & Without antioxidant & $\mathrm{LSD}^{2}$ \\
\hline 0 & 0 & 0.59 & $0 . .59$ & 0.59 & 0.59 & 0.59 & \\
\hline \multirow{3}{*}{15} & 0.01 & 1.99 & 5.10 & 5.90 & \multirow{3}{*}{1.16} & \multirow{3}{*}{5.95} & 1.53 \\
\hline & 0.02 & 0.79 & 1.58 & 3.58 & & & 1.44 \\
\hline & 0.03 & 0.78 & 0.84 & 1.18 & & & 1.63 \\
\hline \multirow{3}{*}{45} & 0.01 & 5.58 & 7.00 & 11.08 & \multirow{3}{*}{1.98} & \multirow{3}{*}{8.74} & 1.25 \\
\hline & 0.02 & 1.64 & 4.34 & 5.18 & & & 1.51 \\
\hline & 0.03 & 0.82 & 1.92 & 2.78 & & & 1.54 \\
\hline \multirow{3}{*}{75} & 0.01 & 6.38 & 7.20 & 11.20 & \multirow{3}{*}{3.18} & \multirow{3}{*}{11.20} & 1.65 \\
\hline & 0.02 & 2.74 & 5.18 & 9.50 & & & 2.24 \\
\hline & 0.03 & 1.50 & 1.58 & 2.78 & & & 2.07 \\
\hline LSD Value & & 1.59 & 1.88 & 2.74 & 1.18 & 2.56 & \\
\hline
\end{tabular}

${ }^{1}$ BHT, butylated hydroxytoluene, ${ }^{2} \mathrm{LSD}$, least significant difference, if the means differ by the LSD number the values are significantly different. 
Table 8. Effect of purified lignans on the thiobarbituric acid (TBA) values of flaxseed oil stored at 65 and $25^{\circ} \mathrm{C}$

\begin{tabular}{|c|c|c|c|c|c|c|}
\hline & & & TB & (mg/ MDA/kg & oil) & \\
\hline $\begin{array}{l}\text { Days } \\
65^{\circ} \mathrm{C}\end{array}$ & Antioxidant (\%) & $\mathrm{LRSO}^{1}$ & $\mathrm{LUSO}^{2}$ & $\operatorname{BHT}^{3}(0.02 \%)$ & Without antioxidant & $\mathrm{LSD}^{4}$ \\
\hline 0 & 0 & 2.52 & 2.52 & 2.52 & 2.52 & \\
\hline & 0.01 & 4.05 & 4.09 & & & 1.46 \\
\hline 4 & 0.02 & 3.45 & 3.80 & 3.19 & 6.59 & 1.54 \\
\hline & 0.03 & 3.27 & 3.27 & & & 1.52 \\
\hline & 0.01 & 6.90 & 7.50 & & & 2.85 \\
\hline 12 & 0.02 & 5.94 & 6.74 & 6.03 & 11.66 & 3.07 \\
\hline & 0.03 & 4.62 & 6.04 & & & 2.78 \\
\hline & 0.01 & 8.58 & 10.36 & & & 3.64 \\
\hline 16 & 0.02 & 7.26 & 8.56 & 7.88 & 14.68 & 3.18 \\
\hline & 0.03 & 5.08 & 6.12 & & & 3.17 \\
\hline LSD Value & & & & & & \\
\hline & & & TB & (mg/MDA/kg & oil) & \\
\hline $\begin{array}{l}\text { Days } \\
25^{\circ} \mathrm{C}\end{array}$ & Antioxidant (\%) & $\mathrm{LRSO}^{1}$ & LUSO $^{2}$ & $\operatorname{BHT}^{3}(0.02 \%)$ & Without antioxidant & $\mathrm{LSD}^{4}$ \\
\hline 0 & 0 & 2.52 & 2.52 & 2.52 & 2.52 & \\
\hline & 0.01 & 4.78 & 5.33 & & & 1.35 \\
\hline 15 & 0.02 & 4.62 & 4.65 & 4.37 & 6.81 & 1.18 \\
\hline & 0.03 & 2.97 & 3.95 & & & 1.43 \\
\hline & 0.01 & 6.53 & 6.91 & & & 1.58 \\
\hline 45 & 0.02 & 5.23 & 5.93 & 5.88 & 9.59 & 1.84 \\
\hline & 0.03 & 4.63 & 5.67 & & & 1.72 \\
\hline & 0.01 & 7.04 & 7.64 & & & 1.63 \\
\hline 75 & 0.02 & 6.19 & 6.49 & 6.24 & 11.38 & 2.05 \\
\hline & 0.03 & 5.21 & 5.98 & & & 1.89 \\
\hline LSD Value & & 2.18 & 1.57 & 1.09 & 2.84 & \\
\hline
\end{tabular}

${ }^{1}$ LRSO, Lignin extracts from roasted sesame seeds, ${ }^{2}$ LUSO, Lignin extracts from unroasted sesame seeds, ${ }^{3} \mathrm{BHT}$, butylated hydroxytoluene, ${ }^{4} \mathrm{LSD}$, least significant difference, if the means differ by the LSD number the values are significantly different.

Table 9. Effect of purified lignans on the thiobarbituric acid (TBA) values of flaxseed oil stored at 65 and $25^{\circ} \mathrm{C}$

\begin{tabular}{|c|c|c|c|c|c|c|c|}
\hline \multirow{2}{*}{ Days $65^{\circ} \mathrm{C}$} & \multirow[b]{2}{*}{ Antioxidant (\%) } & \multicolumn{6}{|c|}{ TBA (mg/ MDA/kg oil) } \\
\hline & & Sesamol & Sesamin & Sesamolin & $\operatorname{BHT}^{1}(0.02 \%)$ & Without antioxidant & $\mathrm{LSD}^{2}$ \\
\hline 0 & 0 & 2.52 & 2.52 & 2.52 & 2.52 & 2.52 & \\
\hline \multirow{3}{*}{4} & 0.01 & 3.38 & 4.78 & 4.29 & \multirow{3}{*}{3.19} & \multirow{3}{*}{6.59} & 1.65 \\
\hline & 0.02 & 3.09 & 3.33 & 4.03 & & & 1.48 \\
\hline & 0.03 & 2.76 & 3.21 & 3.69 & & & 1.73 \\
\hline \multirow{3}{*}{12} & 0.01 & 6.37 & 6.25 & 7.47 & \multirow{3}{*}{6.03} & \multirow{3}{*}{11.66} & 2.39 \\
\hline & 0.02 & 5.86 & 6.23 & 6.47 & & & 2.52 \\
\hline & 0.03 & 5.36 & 5.56 & 5.86 & & & 2.71 \\
\hline \multirow{3}{*}{16} & 0.01 & 6.45 & 6.54 & 7.59 & \multirow{3}{*}{7.88} & \multirow{3}{*}{14.68} & 2.49 \\
\hline & 0.02 & 6.39 & 6.49 & 6.98 & & & 2.77 \\
\hline & 0.03 & 4.83 & 6.12 & 6.44 & & & 2.65 \\
\hline \multirow[t]{2}{*}{ LSD Value } & & 2.68 & 2.75 & 4.07 & 2.63 & 4.78 & \\
\hline & & \multicolumn{6}{|c|}{ TBA (mg/ MDA/kg oil) } \\
\hline Days $25^{\circ} \mathrm{C}$ & Antioxidant (\%) & Sesamol & Sesamin & Sesamolin & $\mathrm{BHT}^{1}(0.02 \%)$ & Without antioxidant & $\mathrm{LSD}^{2}$ \\
\hline 0 & 0 & 2.52 & 2.52 & 2.52 & 2.52 & 2.52 & \\
\hline \multirow{3}{*}{15} & 0.01 & 3.85 & 4.78 & 5.76 & \multirow{3}{*}{4.37} & \multirow{3}{*}{6.81} & 1.47 \\
\hline & 0.02 & 3.69 & 4.49 & 5.07 & & & 1.52 \\
\hline & 0.03 & 1.67 & 3.48 & 4.16 & & & 2.35 \\
\hline \multirow{3}{*}{45} & 0.01 & 5.47 & 6.25 & 7.11 & \multirow{3}{*}{5.88} & \multirow{3}{*}{9.59} & 1.71 \\
\hline & 0.02 & 5.19 & 5.89 & 6.25 & & & 2.45 \\
\hline & 0.03 & 3.20 & 4.97 & 5.80 & & & 2.37 \\
\hline \multirow{3}{*}{75} & 0.01 & 5.98 & 7.27 & 8.91 & \multirow{3}{*}{6.24} & \multirow{3}{*}{11.38} & 2.37 \\
\hline & 0.02 & 5.73 & 6.28 & 6.33 & & & 2.18 \\
\hline & 0.03 & 5.20 & 5.89 & 5.99 & & & 2.54 \\
\hline LSD Value & & 2.16 & 2.09 & 1.86 & 1.09 & 2.84 & \\
\hline
\end{tabular}

${ }^{1}$ BHT, butylated hydroxytoluene, ${ }^{2}$ LSD, least significant difference, if the means differ by the LSD number the values are significantly different. 
and sesamolin respectively.

The TBA values of flaxseed oil treated with LRSO and LUSO and with individual sesame lignans is tabulated in Tables 8 and 9. A similar trend is seen in TBA values in flaxseed oil as with sunflower oil. In Table 8, LRSO samples consistently showed lower TBA values than LUSO and control samples at concentrations of 0.02 and $0.03 \%$ at days 12 and 16 at $65^{\circ} \mathrm{C}$ and days 45 and 75 at $25^{\circ} \mathrm{C}$. LRSO samples were similar to the BHT treated samples, though not always statistically different. In Table 9, at $65^{\circ} \mathrm{C}$ and at day 16 , flaxseed oil samples treated with sesamol, sesamin and sesamolin showed lower TBA values than samples treated with BHT. Samples with sesamol showed the lowest TBA values of all lignans tested at these conditions. At $25^{\circ} \mathrm{C}$, a similar trend is seen with samples containing sesamol having the lowest TBA values as compared to the other treatments, while samples containing sesamin and sesamolin were only effective at $0.03 \%$. Since sesame lignans are considered natural, there is no use level limit and may be used in oils at concentrations of $0.03 \%$.

\section{Discussion}

This study compared the antioxidant activity of crude lignans extracted from roasted and unroasted sesame seed oil in unsaturated food oils. Additionally, the antioxidant activity of sesamin, sesamolin and sesamol purified from roasted sesame seed oil was also investigated in the same oils.

The PV was used to measure the formation of peroxides, which are primary oxidation products. Peroxide formation during lipid storage is initially low, then increases over the storage time then decreases. Peroxides are unstable and will react to form secondary oxidation products such as aldehydes. Since the peroxides decrease over time the PV assay should not be used as the sole assay to judge the rancidity of oils. Therefore, the TBA assay was also used in this study to test for the secondary oxidation products.

The level of peroxide values that gives a perceptible rancid taste in products varies depending on the type of oils. Soybean oils that have peroxide values of 1-5 meq $\mathrm{O}_{2} / \mathrm{kg}$ oil, 5-10 meq $\mathrm{O}_{2} / \mathrm{kg}$ oil and $>10$ meq $\mathrm{O}_{2} / \mathrm{kg}$ oil are considered as slightly, moderately and highly oxidized, respectively (AOCS, 1997; Nielsen, 2003). According to Gunstone (1996), refined vegetable oils should have a peroxide value below $1 \mathrm{meq} \mathrm{O}_{2} / \mathrm{kg}$ oil, and a rancid taste becomes perceptible at approximately $10 \mathrm{meq} \mathrm{O}_{2} / \mathrm{kg}$ oil. It has been reported that a rancid taste is noticeable in many oils that have peroxide values between 20 and 40 meq $\mathrm{O}_{2} / \mathrm{kg}$ oil (Kirk and Sawyer, 1991; O'Brien, 1998). If the value of $>40$ meq $\mathrm{O}_{2} / \mathrm{kg}$ oil is used to denote noticeable rancidity, the control sunflower oil sample without any antioxidant was rancid at day 12 at $65^{\circ} \mathrm{C}$ (Table 2). Sunflower oil was rancid at day 12 at $65^{\circ} \mathrm{C}$ with $0.01 \%$ of LUSO but samples containing LRSO were not. Samples containing $0.02 \%$ LRSO and BHT were not rancid at day 16 . Sunflower oil containing sesamol and BHT was not rancid at day 16 at $65^{\circ} \mathrm{C}$ (Table 3). But samples containing $0.01 \%$ sesamolin were rancid at day 12 and samples containing $0.01 \%$ sesamin were rancid at day 16. Therefore, sesamol was as effective as BHT as an antioxidant.

A similar trend is seen in flaxseed oil, where the control was rancid at day 16 at $65^{\circ} \mathrm{C}$ but the BHT treated sample showed no rancidity (Tables 6 and 7). LRSO was more effective than LUSO since samples did not show rancidity until day 16 at $0.01 \%$ at $65^{\circ} \mathrm{C}$ (Table 6). Samples containing sesamolin at $0.01 \%$ were the only samples with added antioxidant showing rancidity at day 16 at $65^{\circ} \mathrm{C}$ (Table 7). Therefore, sesamol and sesamin at each concentration tested were effective as antioxidants in flaxseed oil.

In general, the oxidation of sunflower and flaxseed oil progressed rapidly without any antioxidant at $65^{\circ} \mathrm{C}$ as compared to at $25^{\circ} \mathrm{C}$ as seen in the control samples. Additionally, lignan extracts from RSO showed higher antioxidant activity then lignan extracts from USO. BHT was used at $0.02 \%$ as a positive control. In general, the LRSO samples showed lower PV values than the BHT treated samples when used at $0.03 \%$ while the LUSO samples showed similar or lower PV values to BHT treated samples.

Our results are similar to a study by Hussain et al. (2018) who showed that methanolic extracts of unroasted sesame oil had antioxidant activity in sunflower oil. In their study, PVs for the sunflower oil control was greater than 15 at 20 days of storage at $25^{\circ} \mathrm{C}$ while samples containing the extract showed PVs less than 5. In the same study, TBA values of control oil were greater than 0.3 at $25^{\circ} \mathrm{C}$ at 30 days while the TBA values of samples treated with extract were lower, approximately 0.25 . They also showed that the antioxidant activity of the extracts was present in baked muffins containing the oil.

Another study investigated the antioxidant activity of crude methanol extracts of sesame oil added to olive, sunflower, soybean or corn oil. The extracts were added to the oils which were then stored oil at $100^{\circ} \mathrm{C}$ over 8 days (Konsoula and Kiakipoulou-Kyriakides, 2010) The oxidative deterioration of the oils progressed rapidly with PVs rising to over 200. With the addition of the methanol extracts the PVs ranged from 40 to 80 , showing that the crude methanol extracts exhibited antioxidant activity. 
There are few studies that investigated the antioxidant activity of the individual sesamin, sesamolin and sesamol. Sesamol is formed from the breakdown of sesamolin which occurs during the roasting of sesame seeds. This lignin has reported antioxidant activity that is higher than sesamin and sesamolin (Dar and Arumugam, 2013). Lee and Choe (2006) purified sesamin, sesamol and sesamolin from roasted sesame seed oil. They investigated the antioxidant activities of these lignans and alpha-tocopherol on the oxidation of methyl linoleate (ML) at $60^{\circ} \mathrm{C}$ over $18 \mathrm{hrs}$ at concentrations of $0.005,0.01$ and $0.02 \%$. At $18 \mathrm{hrs}$, there was a dose-dependent antioxidant activity of the antioxidants with the highest concentration showing the least oxidation. Sesamol showed significantly higher antioxidant activity compared to the other lignans and also to alpha-tocopherol. The order of antioxidant activity was sesamol, followed by alpha-tocopherol, sesamin and sesamolin. This is similar to our findings were sesamol showed the highest antioxidant activity compared to sesamin and sesamolin and at a concentration of $0.03 \%$, the antioxidant activity was greater than BHT which was used at the legal limit of $0.02 \%$.

\section{Conclusion}

Lignan extracts from RSO were as effective as BHT as antioxidants in sunflower and flaxseed oil at concentrations of $0.02 \%$ and of the purified lignans, sesamol showed the highest antioxidant activity. The results of this study revealed that sesame lignans can be easily extracted from roasted sesame seed oil and added to food oils to retard rancidity. Sesame lignans are considered natural antioxidants and may be favored over synthetic antioxidants such as BHT and BHA as ingredients in oils. The oxidation of sunflower and flaxseed oils was investigated in this study, but other unsaturated oils such as olive, safflower and canola may also benefit from the addition of sesame lignans. It is recommended that the extraction of lignans from roasted sesame seed oil be investigated on a pilot scale to determine if the use of sesame lignans as antioxidants is economical for food use.

\section{Conflicts of Interest}

The authors declare no conflicts of interest.

\section{Acknowledgement}

This project was partially supported by the Utah State University Utah Agricultural Experiment Station and approved as journal paper number 9259 .

\section{References}

AOCS. (1997). Official and recommended practices of the American Oil Chemists' Society. $5^{\text {th }}$ ed. Champaign, Illinois, USA: AOCS Press.

AOCS. (2001). Surplus Method Cd 8-53, Peroxide value, acetic acid - chloroform method. Urbana IL, USA: AOCS Press.

AOCS. (2017). Official Method Cd 19-90, 2Thiobarbituric acid value, direct method. Urbana IL, USA: AOCS Press.

Basturk, A., Ceylan, M.M., Cavus, M., Boran, G. and Javidipour, I. (2017). Effects of some herbal extracts on oxidative stability of corn oil under accelerated oxidation conditions in comparison with some commonly used antioxidants. LWT-Food Science and Technology, 89, 358-364. https:// doi.org/10.1016/j.lwt.2017.11.005

Dar, A.A. and Arumugam, N. (2013). Lignans of sesame: purification methods, biological activities and biosynthesis - a review. Bioorganic Chemistry, 50, 1-10. https://doi.org/10.1016/ j.bioorg.2013.06.009

Hemalatha, G.S. (2007). Sesame lignans enhance the thermal stability of edible vegetable oils. Food Chemistry, 105(3), 1076-1085. https:// doi.org/10.1016/j.foodchem.2007.05.023

Gunstone, R. (1996). Fatty acid and lipid chemistry. New York, Chapman and Hall. https:// doi.org/10.1007/978-1-4615-4131-8

Hussain, S.A., Hameed, A., Ajmal, I., Nosheen, S., Suleria, H.A.R. and Song, Y. (2018). Effects of sesame seed extract as natural antioxidant on the oxidative stability of sunflower oil. Journal of Food Science and Technology, 55(10), 4099-4110. https:// doi.org/10.1007/s13197-018-3336-2

Kirk, R.S. and Sawyer, R. (1991). Pearson's composition and analysis of foods. $9^{\text {th }}$ ed. New York: Longman, Wiley.

Konsoula, Z. and Kiakipoulou-Kyriakides, M. (2010). Effect of endogenous antioxidants of sesame seeds and sesame oil to the thermal stability of edible vegetable oils. LWT-Food Science and Technology, 43(9), 1379-1386. https://doi.org/10.1016/ j.lwt.2010.04.016

Lee, J. and Choe, E. (2006). Extraction of lignan compounds from roasted sesame oil and their effects on the autoxidation of methyl linoleate. Food Chemistry and Toxicology, 71(7), 430-436. https:// doi.org/10.1111/j.1750-3841.2006.00137.x

Mohanan, A., Nickerso, M.T. and Ghost, S. (2018). Oxidative stability of flaxseed oil: effect of hydrophilic, hydrophobic and intermediate polarity 
antioxidant. Food Chemistry, 266, 524-523. https://

doi.org/10.1016/j.foodchem.2018.05.117

Mohdaly, A.A.A., Sarhan, M.A., Mahmoud, A., Ramad, M.F. and Smetanska, I. (2010). Antioxidant efficacy of potato peels and sugar beet pulp extracts in vegetable oil protection. Food Chemistry, 123(4), 1019-1026. https://doi.org/10.1016/ j.foodchem.2010.05.054

Nielsen, S.S. (2003). Food Analysis. 3rd ed. New York: Kluwer Academic/Plenum Publishers.

O'Brien, R.D. (1998). Fats and oils, formulating and processing for applications. Lancaster, PA, USA: Technomic Publishing.

Poiana, M.A. (2012). Enhancing oxidative stability of sunflower oil during convective and microwave heating using grape seed extract. International Journal of Molecular Sciences, 13(7), 9240-9259. https://doi.org/10.3390/ijms13079240

Reshma, M.V., Balachandran, C., Arumughan, C., Sundersan, A., Sukumaran, D., Thomas, S. and Saritha, S.S. (2010). Extraction, separation and characterization of sesame oil lignin for nutraceutical applications. Food Chemistry, 120(4), 1041-1046. https://doi.org/10.1016/j.foodchem.2009.11.047 